\title{
CONSTRUCTING IRREDUCIBLE POLYNOMIALS WITH PRESCRIBED LEVEL CURVES OVER FINITE FIELDS
}

\author{
MIHAI CARAGIU
}

(Received 14 January 2001 and in revised form 28 March 2001)

\begin{abstract}
We use Eisenstein's irreducibility criterion to prove that there exists an absolutely irreducible polynomial $P(X, Y) \in G F(q)[X, Y]$ with coefficients in the finite field $G F(q)$ with $q$ elements, with prescribed level curves $X_{\mathcal{C}}:=\left\{(x, y) \in G F(q)^{2} \mid P(x, y)=c\right\}$.

2000 Mathematics Subject Classification. 11T06.
\end{abstract}

1. Introduction. Let $G F(q)$ be the finite field with $q$ elements. Assume that for any $c \in G F(q)$, a subset $X_{c}$ (possibly empty) of the finite affine plane $G F(q)^{2}$ is given, such that $X_{c} \cap X_{d} \neq \varnothing$ for any $c \neq d$ and

$$
G F(q)^{2}=\bigcup_{c \in G F(q)} X_{c}
$$

In this paper, we use Eisenstein's irreducibility criterion to build absolutely irreducible polynomials

$$
P(X, Y) \in G F(q)[X, Y]
$$

such that for any $c \in G F(q)$ the level curve $\left\{(x, y) \in G F(q)^{2} \mid P(x, y)=0\right\}$ coincides with $X_{c}$. Note that $P(X, Y) \in G F(q)[X, Y]$ is called absolutely irreducible if it is irreducible over the algebraic closure of $G F(q)$.

If we define a function $f: G F(q)^{2} \rightarrow G F(q)$ taking a constant value $c$ on the set $X_{c}$ for any $c \in G F(q)$, it is easy to see that this is equivalent to the fact that there exists an absolutely irreducible polynomial which interpolates the function $f$.

It is of course well known that there exists a polynomial that interpolates the function $f$ (see [3, Section 7.5] for a general discussion on this topic). Thus, our result can be viewed as a stronger version of this basic fact, going back to Weber [4].

The basic facts about bivariate polynomial interpolation over finite fields that we will need are summarized in the following theorem.

THEOREM 1.1. Any function $f: G F(q)^{2} \rightarrow G F(q)$ can be interpolated by some polynomial in two variables. Moreover, there exists a unique polynomial $F(X, Y) \in G F(q)[X, Y]$ of degree less than $q$ in both $X$ and $Y$ that interpolates the function $f$, that is, satisfying $F(a, b)=f(a, b)$ for any $(a, b) \in G F(q)^{2}$. Also, any two interpolating polynomials for $f$ are congruent modulo the ideal of $G F(q)[X, Y]$ generated by $X^{q}-X$ and $Y^{q}-Y$. 
Our main result is the following theorem.

THEOREM 1.2. Let $f: G F(q)^{2} \rightarrow G F(q)$ be a function. Then there exists an absolutely irreducible polynomial $P(X, Y) \in G F(q)[X, Y]$ that interpolates the function $f$.

2. Proof of the main result. Let $f: G F(q)^{2} \rightarrow G F(q)$ be an arbitrary function. By Theorem 1.1, there exists a unique interpolating polynomial $H(X, Y) \in G F(q)[X, Y]$ for $f$, of degree at most $q-1$ in both $X$ and $Y$. We order $H(X, Y)$ in terms of the powers of $Y$

$$
H(X, Y)=c_{0}(X)+c_{1}(X) Y+\cdots+c_{q-1}(X) Y^{q-1},
$$

where $c_{0}(X), c_{1}(X), \ldots, c_{q-1}(X) \in G F(q)[X, Y]$ are of degree at most $q-1$.

Clearly, if we add $Y^{q}-Y$ to $H(X, Y)$, we still get an interpolating polynomial for $f$, say $K(X, Y)$, that is, monic in $Y$. Thus, it will be perfectly legitimate to start with an interpolating polynomial of the form

$$
K(X, Y)=Y^{q}+d_{q-1}(X) Y^{q-1}+\cdots+d_{1}(X) Y+d_{0}(X),
$$

where $d_{0}(X), d_{1}(X), \ldots, d_{q-1}(X) \in G F(q)[X, Y]$ are of degree at most $q-1$.

It is well known (see [3, Corollary 2.11]) that there are irreducible polynomials of any degree over a finite field $G F(q)$. Fix such an irreducible polynomial $h(X) \in G F(q)[X]$ of degree 2. Clearly $h(X)$ has two roots in the algebraic closure of $G F(q)$, each of them generating the quadratic extension of $G F(q)$. Let $\alpha$ be a root of $h(X)$ in $\overline{G F(q)}$, the algebraic closure of $G F(q)$.

Our construction is based on replacing each polynomial coefficient $d_{i}(X)$ of (2.2) with a polynomial of the form

$$
e_{i}(X)=d_{i}(X)+\left(X^{q}-X\right) u_{i}(X)
$$

where $u_{i}(X) \in G F(q)[X]$, such that each $e_{i}(X)$ is divisible by $h(X)$ for $i=0, \ldots, q-1$, while $e_{0}(X)$ is not divisible by $h(X)^{2}$. Clearly, the polynomial $F(X, Y)$ we get by performing these replacements

$$
F(X, Y)=Y^{q}+e_{q-1}(X) Y^{q-1}+\cdots+e_{1}(X) Y+e_{0}(X)
$$

will still be an interpolating polynomial for $f$, by Theorem 1.1. We will then see that $F(X, Y)$ follows to be absolutely irreducible.

We prove that for some choice of $u_{i}(X) \in G F(q)[X]$ in (2.3), $e_{i}(X)$ is divisible by $h(X)$, that is,

$$
d_{i}(X)+\left(X^{q}-X\right) u_{i}(X) \equiv 0 \quad(\bmod h(X))
$$

is solvable. Indeed, from the way we defined $h(X), X^{q}-X$ is relatively prime to $h(X)$. Thus, (2.5) is a linear congruence modulo $h(X)$ in the Euclidean ring $G F(q)[X]$ in which the coefficient $X^{q}-X$ of the unknown $u_{i}(X)$ is relatively prime to the modulus $h(X)$. This being the case, a solution $u_{i}(X)$ of (2.5) exists, and is uniquely determined up to a multiple of $h(X)$. It follows that we can select a solution $u_{i}(X)$ of (2.5) which is 
a polynomial of degree one. This will completely take care of the cases $i=1, \ldots, q-1$. For the special case $i=0$ we are looking for a solution $u_{0}(X)$ of (2.5) satisfying the additional requirement

$$
d_{0}(X)+\left(X^{q}-X\right) u_{0}(X) \not \equiv 0 \quad\left(\bmod h(X)^{2}\right)
$$

This can be done as follows. If the solution $u_{0}(X)$ of the $i=0$ case of (2.5) already satisfies (2.6) there is nothing to prove. Otherwise, if $u_{0}(X)$ satisfies

$$
d_{0}(X)+\left(X^{q}-X\right) u_{0}(X) \equiv 0 \quad\left(\bmod h(X)^{2}\right),
$$

just replace $u_{0}(X)$ with $u_{0}(X)+h(X)$. This last polynomial will satisfy both (2.5) and (2.6).

The last step in our proof will consist in showing that the polynomial $F(X, Y)$ constructed above is absolutely irreducible.

The key ingredient of this last step is Eisenstein's irreducibility criterion (see [2, Theorem 6.15]), to the effect that if $P(X)=\gamma_{n} X^{n}+\gamma_{n-1} X^{n-1}+\cdots+\gamma_{1} X+\gamma_{0}$ is a polynomial with coefficients in some unique factorization domain $\mathbb{R}$, if we can find some irreducible element $p \in \mathbb{R}$ which divides $\gamma_{0}, \ldots, \gamma_{n-1}$, does not divide $\gamma_{n}$, while $p^{2}$ does not divide $\gamma_{0}$, then $P(X)$ is an irreducible element of $R[X]$.

We view $F(X, Y)$ as a (monic) polynomial in $Y$ with coefficients in the unique factorization domain $\overline{G F(q)}[X]$, that is, $F(X, Y) \in(\overline{G F(q)}[X])[Y]$.

Pick up the irreducible

$$
p(X):=X-\alpha \in \overline{G F(q)}[X] .
$$

Since $\alpha$ is a root of $h(X)$, by the way we constructed $F(X, Y)$ it follows that $p(X)$ divides the polynomial coefficients $e_{0}(X), e_{1}(X), \ldots, e_{q-1}(X) \in G F(q)[X]$ and $p(X)^{2}$ does not divide the free coefficient $e_{0}(X)$. Also, the coefficient of the highest power of $Y$ in (2.4) is 1 . Thus, we can apply now Eisenstein's criterion to conclude that $F(X, Y)$ is an irreducible element of the polynomial ring $(\overline{G F(q)}[X])[Y] \cong \overline{G F(q)}[X, Y]$. In other words, the interpolating polynomial $F(X, Y)$ for $f$ is absolutely irreducible. This concludes the proof of our main theorem.

By our construction, the degrees of the polynomial coefficients $e_{1}(X), \ldots, e_{q-1}(X)$ of $F(X, Y)$ are at most $q+1$, the degree of $e_{0}(X)$ is at most $q+2$, while $F(X, Y)$ is monic of degree $q$ in $Y$.

Theorem 1.2 may be seen as a useful tool in the theory of curves over finite fields, since it allows a fairly elementary and efficient construction of equations of absolutely irreducible plane curves over $G F(q)$ with a given set $Z \subset G F(q)^{2}$ of $G F(q)$-rational points (we may, for example, apply our construction to the special case in which the level curves are $X_{0}=Z, X_{1}=G F(q)^{2} \backslash Z$, and $X_{c}=\varnothing$ for any $\left.c \in G F(q) \backslash\{0,1\}\right)$. Finally, our interpolation result (with a construction based on a different method, though less direct) still holds true for the case of more than two variables (the proof of this will appear in [1]). 


\section{REFERENCES}

[1] M. Caragiu, Multivariate interpolation by absolutely irreducible polynomials over finite fields, to appear in Rev. Roumaine Math. Pures Appl.

[2] T. W. Hungerford, Algebra, Graduate Texts in Mathematics, vol. 73, Springer-Verlag, New York, 1980, reprint of the 1974 original. MR 82a:00006. Zbl 442.00002.

[3] R. Lidl and H. Niederreiter, Finite Fields, 2nd ed., Encyclopedia of Mathematics and its Applications, vol. 20, Cambridge University Press, Cambridge, 1997. MR 97i:11115. Zbl 866.11069.

[4] H. Weber, Lehrbuch der Algebra, vol. 2, Vieweg \& Sohn, Braunschweig, 1899.

Mihai CARAgiu: The InSTITUTe of MAThematics at Bucharest, P.O. Box 1-764, RO-70700, ROMANIA

Current address: Department of MATHEMATICS, OHIO NORTHERn UNIVERSity, ADA, OH 45810, USA

E-mail address: m-caragiu1@onu. edu 


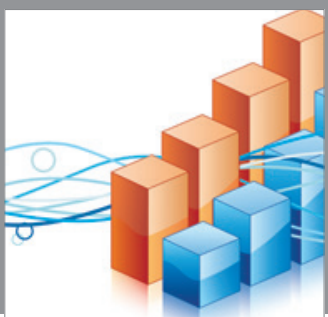

Advances in

Operations Research

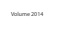

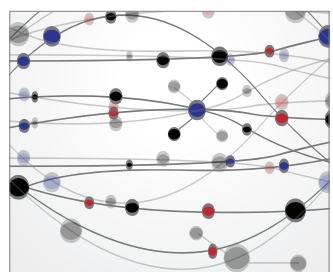

\section{The Scientific} World Journal
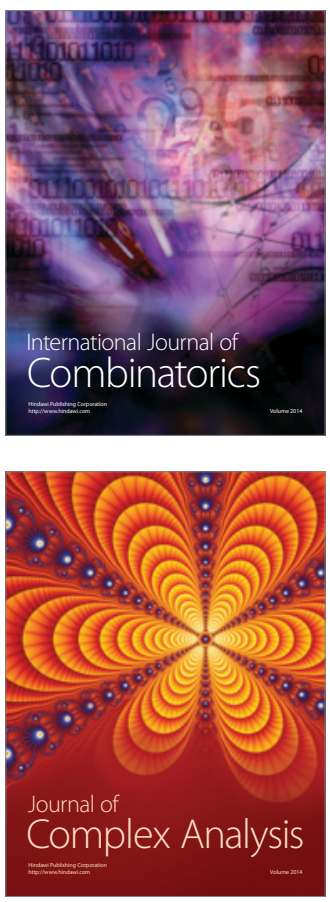

International Journal of

Mathematics and

Mathematical

Sciences
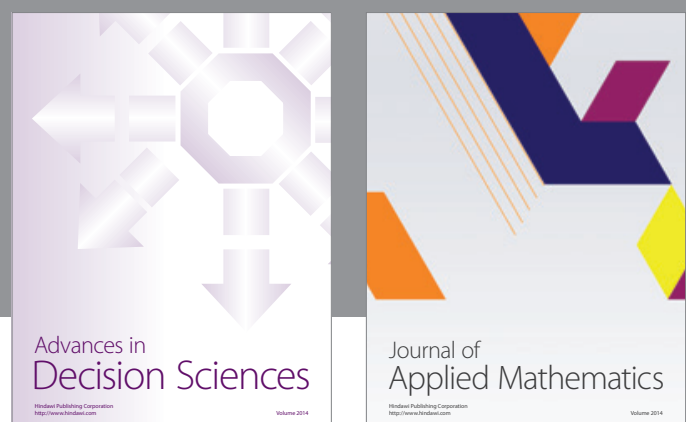

Journal of

Applied Mathematics
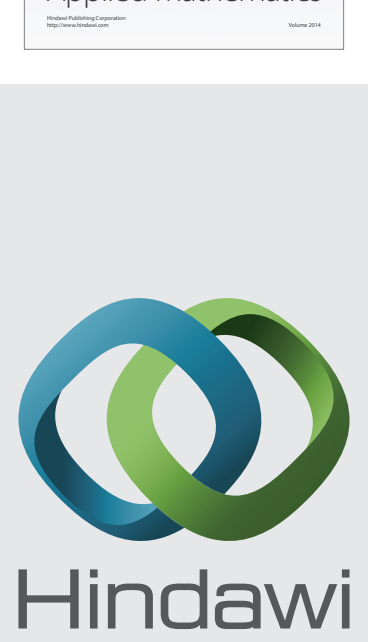

Submit your manuscripts at http://www.hindawi.com
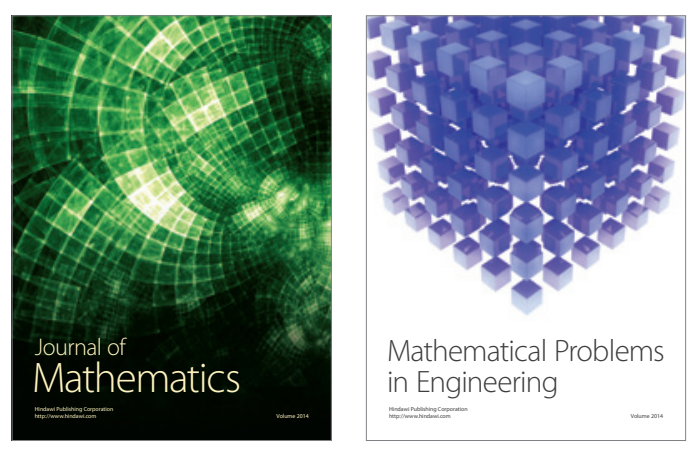

Mathematical Problems in Engineering
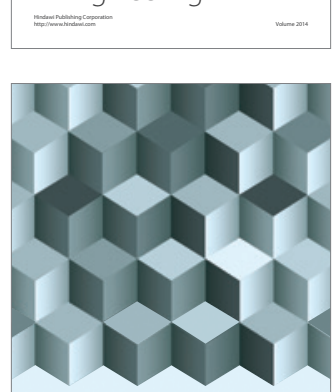

Journal of

Function Spaces
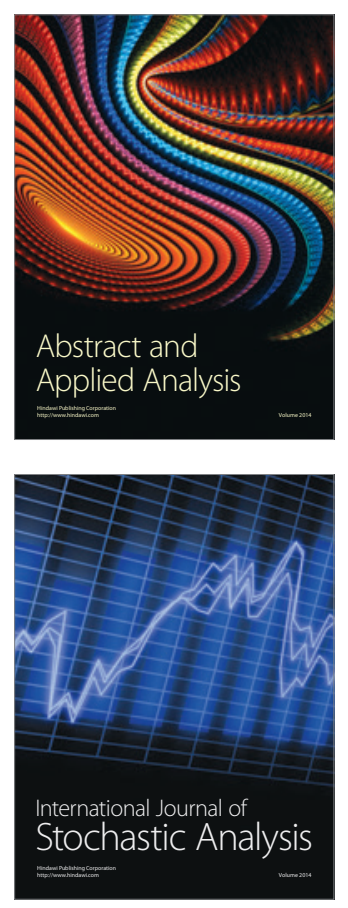

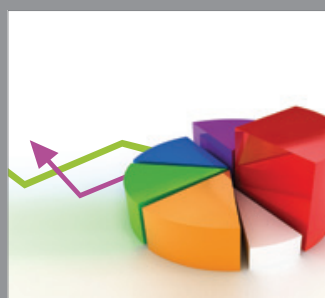

ournal of

Probability and Statistics

Promensencen
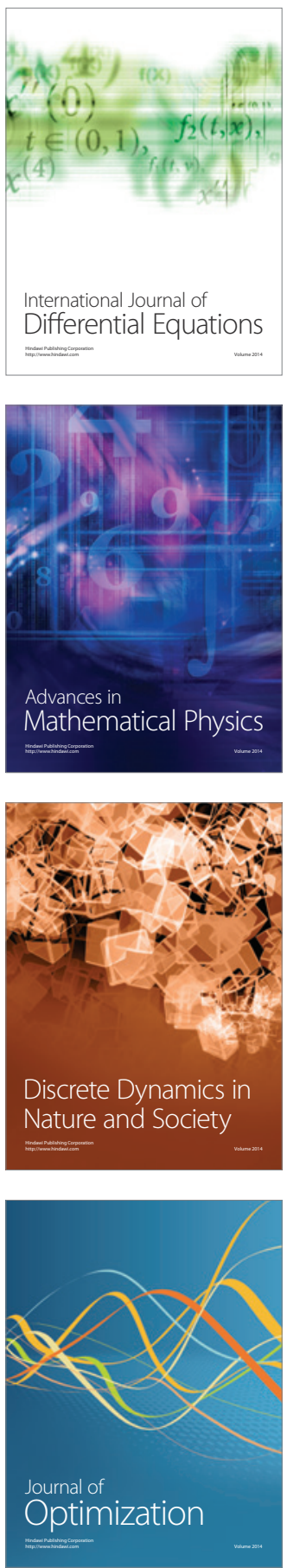